\title{
Quantum Temporal Imaging
}

\author{
Mankei Tsang and Demetri Psaltis \\ Department of Electrical Engineering, California Institute of Technology, \\ Pasadena, California 91125 \\ mankei@sunoptics.caltech.edu
}

\begin{abstract}
The concept of quantum temporal imaging is proposed to manipulate the temporal correlation of entangled photons. In particular, we show that time correlation and anticorrelation can be converted to each other using quantum temporal imaging.

(C) 2006 Optical Society of America

OCIS codes: (270.4180) Multiphoton processes; (270.5290) Photon statistics.
\end{abstract}

\section{Introduction}

Conventional wisdom suggests that one can only manipulate the temporal correlation of two entangled photons by engineering the photon pair generation process. For example, it is generally believed that a long pump pulse is required to generate time correlation, while an ultrashort pump pulse and extended phase matching conditions are needed to generate time anticorrelation. This means that one is unable to take advantage of the high conversion efficiency offered by an ultrashort pump pulse if time correlation is desired, while one has to depend on the availability of an ultrafast laser and a scrupulously fabricated nonlinear medium in order to generate time anticorrelation. In this paper, we propose the concept of quantum temporal imaging, which allows one to impose arbitrary correlations on two already entangled photons using simple linear optics, thereby permitting much more flexibility in the configuration of photon pair sources. Most significantly, we show that perfect time correlation and perfect time anticorrelation, which are the most useful types of temporal entanglement for quantum-enhanced time measurement applications [1], can be converted to each other using a quantum temporal imaging system.

\section{Theory}

The concept of quantum temporal imaging exploits the analogy between diffraction and group-velocity dispersion, as well as that between lensing and temporal phase modulation. The optical propagation of two photons in two modes under these effects is described by

$$
\left[\frac{}{z_{j}}+{ }_{1 j}\left(z_{j}\right) \frac{}{t_{j}}\right] \hat{A}_{j}\left(z_{j}, t_{j}\right)=\left[-\frac{i 2 j\left(z_{j}\right)}{2} \frac{2}{t_{j}^{2}}+i k_{0} \quad n_{j}\left(z_{j}, t_{j}\right)\right] \hat{A}_{j}\left(z_{j}, t_{j}\right), \quad j=1,2
$$

where $\hat{A}_{j}$ is the complex envelope annihilation operator of the $j$ th mode, ${ }_{1 j}$ is the group delay, $2 j$ is the groupvelocity-dispersion coefficient, and $n_{j}$ is the refractive index perturbation, such as that by an electro-optic modulator. The two-photon probability amplitude defined as

$$
\left(z_{1}, t_{1}, z_{2}, t_{2}\right)=\left\langle 0,0\left|\hat{A}_{1}\left(z_{1}, t_{1}\right) \hat{A}_{2}\left(z_{2}, t_{2}\right)\right| 1,1\right\rangle
$$

then obeys a pair of propagation equations,

$$
\begin{aligned}
& {\left[\frac{}{z_{1}}+{ }_{11}\left(z_{1}\right) \frac{}{t_{1}}\right] \quad\left(z_{1}, t_{1}, z_{2}, t_{2}\right)=\left[-\frac{i 21\left(z_{1}\right)}{2} \frac{2}{t_{1}^{2}}+i k_{0} n_{1}\left(z_{1}, t_{1}\right)\right] \quad\left(z_{1}, t_{1}, z_{2}, t_{2}\right)} \\
& {\left[\frac{}{z_{2}}+{ }_{12}\left(z_{2}\right) \frac{}{t_{2}}\right] \quad\left(z_{1}, t_{1}, z_{2}, t_{2}\right)=\left[-\frac{i 22\left(z_{2}\right)}{2} \frac{2}{t_{2}^{2}}+i k_{0} n_{2}\left(z_{2}, t_{2}\right)\right] \quad\left(z_{1}, t_{1}, z_{2}, t_{2}\right) \text {. }}
\end{aligned}
$$

In other words, temporal effects that are classically used to manipulate the optical envelopes [2, 3] can also be used to manipulate the two-photon probability amplitude for quantum optics applications. 


\section{QWB5.pdf}

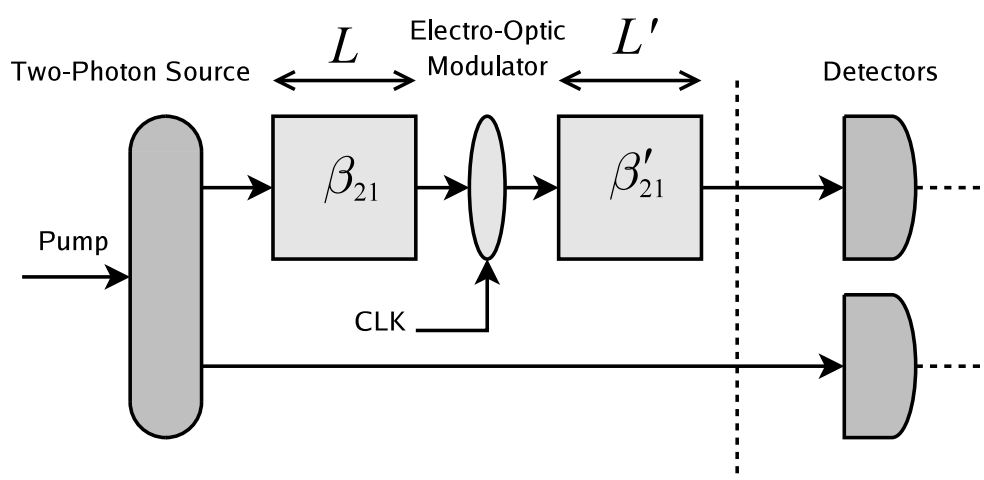

Fig. 1. A quantum temporal imaging system for correlation conversion.

\section{Temporal Correlation Conversion}

Consider the setup depicted in Fig. 1, where a photon in one arm of the two-photon source goes through a single-lens temporal imaging system. If the temporal phase modulation is quadratic, such that the output two-photon amplitude of

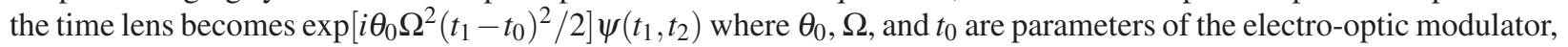
and the temporal lens law is satisfied,

$$
\frac{1}{{ }_{21} L}+\frac{1}{{ }_{21}^{\prime} L^{\prime}}=0^{2}
$$

the two-photon amplitude at the detectors becomes

$$
\text { out }\left(t_{1}, t_{2}\right)=\operatorname{in}\left(\frac{t_{1}-t_{d}}{M}, t_{2}\right), \quad M=-\frac{{ }_{21} L^{\prime}}{{ }_{21} L},
$$

where $t_{d}$ is some time delay and $M$ is the magnification factor. The most interesting case is when $M=-1$, and one of the photons is time-reversed. If the two photons are initially time-correlated, then they become time-anticorrelated at the output, or vice versa. For the specific application of clock synchronization [1], the subclassical arrival-timedifference uncertainty of time-correlated photons can then be converted to a subclassical mean-arrival-time uncertainty, leading to quantum enhancement of clock synchronization accuracy by a factor of $\sqrt{2}$. In practice, the clock can be synchronized with the electro-optic modulator, so that the mean arrival time is controlled by $t_{0}$ and thus the clock. As evident from Eq. (6), any desired correlation can actually be imposed on already entangled photons, by multiplying the original correlation by $1 / M$.

The accuracy of the temporal imaging system is limited by parasitic effects such as higher-order dispersion and phase modulation. An imperfect time lens is likely to be the most detrimental factor in practice, as electro-optic modulators usually produce sinusoidal modulation and a quadratic profile can only be obtained approximately.

\section{Conclusion}

In conclusion, the concept of quantum temporal imaging is proposed to perform temporal correlation conversion of entangled photons, enabling much more flexibility in the choice and configuration of two-photon sources. More complex quantum temporal imaging systems can also be designed using Fourier optics [4], temporal imaging [2, 3], and quantum imaging [5] techniques and are potentially useful for other quantum optics applications.

\section{References}

[1] V. Giovannetti, S. Lloyd, and L. Maccone, "Quantum-enhanced positioning and clock synchronization," Nature (London) 412, 417 (2001).

[2] B. H. Kolner and M. Nazarathy, "Temporal imaging with a time lens," Opt. Lett. 14, 630 (1989).

[3] B. H. Kolner, "Space-time duality and the theory of temporal imaging," IEEE J. Quantum Electron. 30, 1951 (1994).

[4] J. W. Goodman, Introduction to Fourier Optics (McGraw-Hill, Boston, 1996).

[5] A. F. Abouraddy, B. E. A. Saleh, A. V. Sergienko, and M. C. Teich, "Entangled-photon Fourier optics," J. Opt. Soc. Am. B 19, 1174 (2002). 